\title{
Controle da malária no Estado do Maranhão
}

\author{
Malaria control in the State of Maranhão
}

\author{
Antonio Rafael da Silva ${ }^{1}$, José Maurício Carneiro Fernandes ${ }^{2}$, Thiago Alves Rodrigues ${ }^{2}$, \\ Henrique Jorge dos Santos $^{3}$, Nelson Nazareno Miranda Cavalheiro ${ }^{3}$, \\ Milton Cavalcante Guimarães ${ }^{3}$ e Eloísa da Graça do Rosário Gonçalves ${ }^{1}$
}

\begin{abstract}
RESUMO
Este estudo objetiva mostrar os resultados do controle da malária no Estado do Maranhão, com base nos Planos Estaduais de Controle e na série histórica dos registros de malária, no período de 1999 a 2007. A evolução da malária foi analisada comparando-se a incidência parasitária anual (total de casos notificados por 1.000 habitantes) por Região Geográfica, por Unidades Regionais de Saúde e pelos 46 municípios incluídos nos Planos. A análise dos resultados permitiu concluir pela eficácia dos Planos, pois a incidência anual apresentou redução de 10,1 casos para 1,1 casos por 1000 habitantes, uma regressão de 89,1\%, a maior entre todos os estados amazônicos.
\end{abstract}

Palavras-chaves: Malária. Controle. Epidemiologia. Estado do Maranhão.

\section{ABSTRACT}

This study aimed to show the results from malaria control in the State of Maranhão, based on the state's control plans and on the historical series of malaria records over the period from 1999 to 2007. The evolution of malaria cases was analyzed by comparing the annual incidences of parasitism (total number of cases notified per 1,000 inhabitants) according to geographical area, regional healthcare units and the 46 municipalities included in the plans. Analysis of the results allowed the conclusion that the plans were effective, since the annual incidence went down from 10.1 cases to 1.1 cases per 1,000 inhabitants, i.e. a regression of $89.1 \%$, the largest among all the states in the Amazon region.

Key-words: Malaria. Control. Epidemiology. State of Maranhão.

Em 1992, a Organização Mundial da Saúde (OMS) propôs, na Conferência Ministerial sobre Malária, em Amsterdam, uma nova estratégia de controle da doença, enfatizando o controle integrado da malária na rede de serviços de saúde ${ }^{10}$. Seguindo essas diretrizes, o Ministério da Saúde (MS), também, propôs o Programa de Controle Integrado da Malária (PCIM), em substituição ao modelo vertical até então desenvolvido ${ }^{310}$. Esse programa cumpriu, em parte, os objetivos, constatando-se, entre 1995 e 1998, tendência de queda do número de casos da malária que passou de 564.570, no primeiro ano, para 471.892, no segundo ${ }^{6919}$. No final de 1998, a OMS divulgou uma análise da situação epidemiológica da malária no mundo, desenvolvendo a proposta de fazer recuar a malária (Roll Back Malária)²

1. Centro de Referência em Doenças Infecciosas e Parasitárias, Universidade Federal do Maranhão, São Luís, MA. 2. Curso de Medicina, Universidade Federal do Maranhão, São Luís, MA. 3. Secretaria de Estado da Saúde e Programa de Controle da Malária no Estado do Maranhão, São Luís, MA.

Endereço para correspondência: Dr. Antonio Rafael da Silva. Centro de Referência em Doenças Infecciosas e Parasitárias/UFMA. Praça Madre Deus 02, Térreo, 65025-560 São Luís, MA.

Telefax: 5598 3221-0270

e-mail: regionalsbmt@elo.com.br

Recebido para publicação em 20/11/2008

Aceito em 03/04/2009
Para enfrentar uma situação emergencial, em 2000, quando foram registrados 637.472 casos, o MS pôs em prática o Plano de Intensificação das Ações de Controle da Malária (PIACM) ${ }^{4}$. A principal meta era reduzir no prazo de um ano a incidência da malária em 50\%, em 254 municípios de nove Estados da Amazônia Legal, responsáveis por $93,6 \%$ dos casos de malária registrados no país em 1999. Os critérios de inclusão dos municípios foram ser de alto risco, responder por $80 \%$ dos casos de malária no estado, ter transmissão urbana, apresentar índice de malária por Plasmodium falciparum maior ou igual a $20 \%$ e ser capital dos estados ${ }^{46}$.

Em 2003, o PIACM foi substituído pelo Plano Nacional de Controle da Malária (PNCM $)^{8}$ que estendeu suas ações às regiões endêmicas e não-endêmicas e buscou sustentabilidade política para garantir o alcance de metas tais como redução da incidência, das internações e da letalidade em 15\% a cada ano; eliminação da transmissão da malária em áreas urbanas e em capitais até 2006 e manter, sem transmissão, as áreas sem manifestação da doença nos últimos cincos anos.

Na perspectiva do PIACM e do PNCM, o Maranhão desenvolveu seus planos de ação ${ }^{15} 16$. 0 objetivo deste estudo é apresentar os resultados da aplicação dos referidos planos, uma vez que trabalhos recentes sobre malária no Estado do Maranhão foram publicados $^{141718}$. Porém, nenhum com o objetivo de analisar o impacto dos planos de controle, numa perspectiva espaçotemporal. 


\section{MATERIAL E MÉTODOS}

O Maranhão está localizado no hemisfério sul, ocupando a porção ocidental da Região Nordeste do Brasil, tendo como limites: ao norte o Oceano Atlântico, ao sul, o Estado do Tocantins, a leste, o Estado do Piauí e a oeste, os Estados do Pará e Tocantins. Está incluído entre os nove estados da Amazônia Legal. Com área de $331.933,3 \mathrm{~km}^{2}$, população de 6.184 .538 habitantes e densidade demográfica de 18,6 habitantes por $\mathrm{km}^{2}$, é coberto por uma vegetação composta por mata de cocais, campos inundáveis, mangues, cerrado e floresta Amazônica. De clima tropical, é cortado por 12 bacias hidrográficas. Possui 217 municípios, distribuídos em cinco regiões geográficas': norte, na linha litorânea; oeste, seguindo a linha dos grandes rios (Gurupi, Tocantins, Itinga e foz do Araguaia); sul, compreendendo a Chapada das Mangabeiras; centro, abrangendo as bacias do Alto e Baixo Mearim e leste, no curso do Parnaíba (Figura 1). Do ponto de vista estratégico, a Secretaria de Estado da Saúde dividiu o estado em 18 Unidades Regionais de Saúde (URS): Açailândia, Bacabal, Balsas, Barra do Corda, Caxias, Chapadinha, Codó, Imperatriz, Itapecurumirim, Pedreiras, Pinheiro, Presidente Dutra, Rosário, Santa Inês, São Luís, São João dos Patos, Viana e Zé Doca (Figura 1).

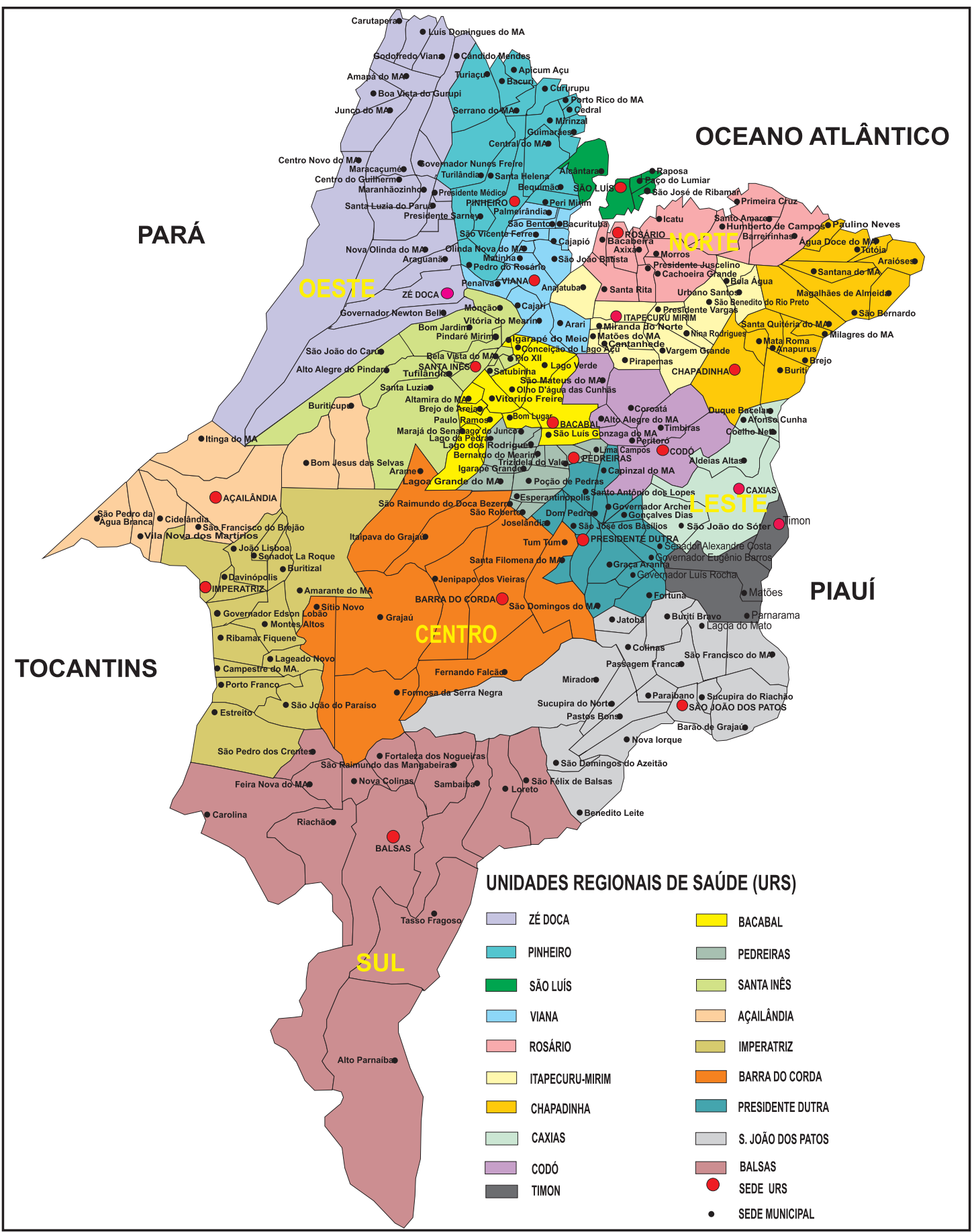

FIGURA 1

Mapa do Maranhão por Regiões Geográficas e Unidades Regionais de Saúde. 
Trata-se de um estudo ecológico, com base nos Planos Estaduais de Controle da Malária e na série histórica dos registros de malária no Maranhão de janeiro de 1999 a dezembro de 2007. Os dados numéricos e as informações sobre a dinâmica de implantação dos Planos foram obtidos no Programa Sivep/Malária, SUS/MS; na Coordenação do Programa de Controle da Malária da Secretaria de Estado da Saúde e no Centro de Referência em Doenças Infecciosas e Parasitárias.

A evolução da malária foi analisada no período, comparando-se a incidência parasitária anual (IPA), total de casos notificados por 1.000 habitantes, nas Regiões Geográficas, nas Unidades Regionais de Saúde (URS) e nos 46 municípios incluídos no PIACM.

\section{RESULTADOS}

Avaliação geral. As principais medidas tomadas pelos Planos Estaduais, com ajuda da Secretaria de Vigilância em Saúde foram criar os Núcleos de Epidemiologia e Controle de Doenças nas 18 URS; acelerar o processo de certificação dos municípios integrantes do Plano e a seguir estendê-lo aos outros municípios; manter pessoal qualificado e comissionado, à disposição dos municípios aos quais serviam; manter os inspetores (denominação dada aos servidores de comando de campo da FUNASA) na direção do controle das endemias nos municípios; expandir a rede de laboratórios; garantir e manter os insumos necessários para o diagnóstico, tratamento e controle vetorial; garantir meios de transporte para manter a vigilância nas localidades distantes com transmissão de malária; treinar a rede de serviços no diagnóstico, tratamento e controle de vetores. As medidas aplicadas não impediram inicialmente, o aumento de 43,2\% na incidência da malária em 2000. A partir desse momento, no entanto, houve redução acentuada na incidência e na morbidade (Figura 2).

Avaliação por região. Em 1999, as regiões norte e oeste apresentaram maior incidência de malária, com IPA de 43.6/1.000 e 8.1/1.000, respectivamente, concentrando $90 \%$ da malária registrada no estado. Quanto às outras regiões - sul, leste e centro - somente esta última apresentava um município com alta incidência (Tabela 1). Constata-se que em 2000, houve aumento na incidência de malária em todas as regiões, seguindose de diminuição significativa e mantida, excetuando o ano de 2004. Na primeira avaliação, ocorrida em 2001, verifica-se que a região norte apresentou índice de redução na incidência de 8,6\%; a região sul de 45,7\%; a região oeste de 53,6\%. As regiões leste e centro apresentaram incremento de 37,5\% e 100\%, respectivamente, em relação aos registros de 1999. Comparandose os anos de 1999 e 2007, observa-se queda da incidência em todas as regiões, havendo a menor variação na região centro $(82,7 \%)$ e a maior na região sul $(97,1 \%)$.

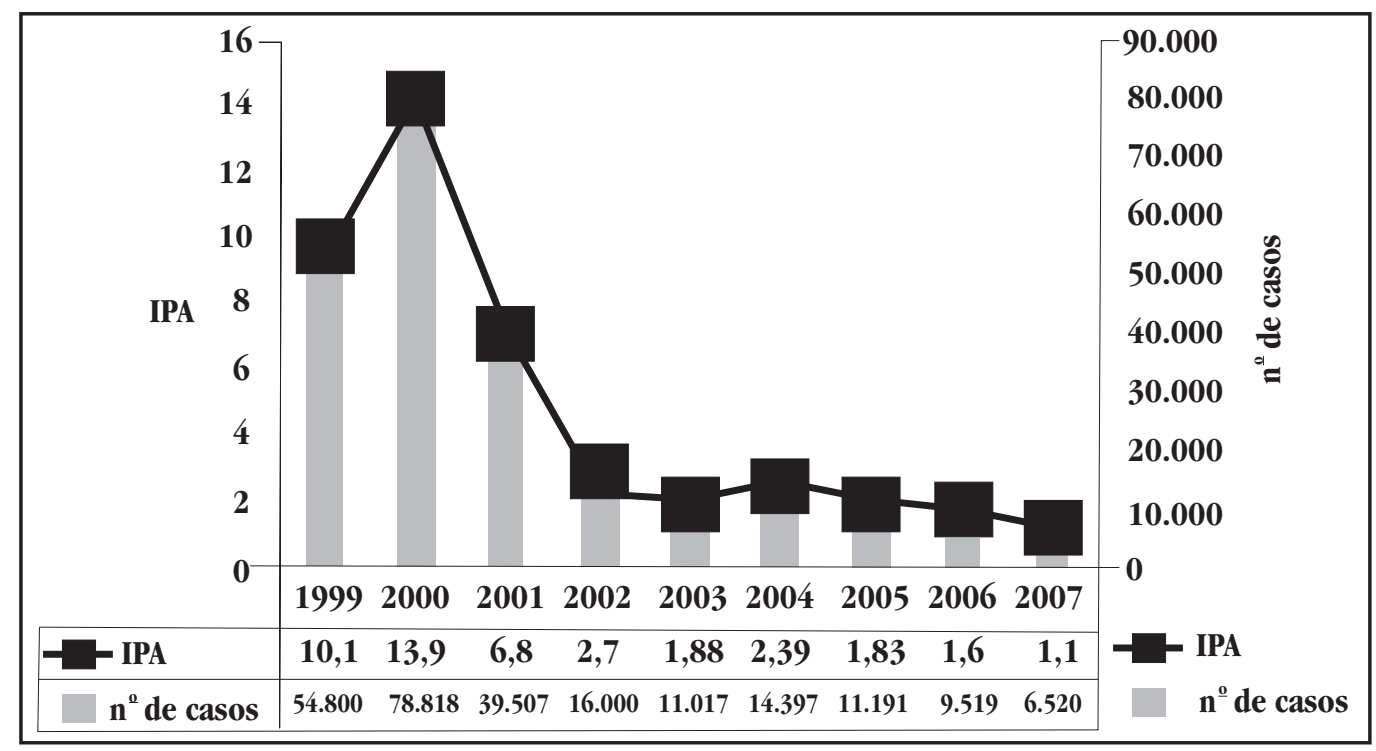

FIGURA 2

Incidência parasitária anual de casos de malária no Estado do Maranhão, 1999 a 2007.

TABELA 1

Incidência parasitária anual da malária por região, Estado do Maranhão, 1999 a 2007.

\begin{tabular}{|c|c|c|c|c|c|c|c|c|c|c|}
\hline \multirow[b]{2}{*}{ Região } & \multicolumn{9}{|c|}{ Ano } & \multirow{2}{*}{$\begin{array}{l}\text { Índice de } \\
\text { redução (\%) }\end{array}$} \\
\hline & 1999 & 2000 & 2001 & 2002 & 2003 & 2004 & 2005 & 2006 & 2007 & \\
\hline Norte & 8,1 & 11,4 & 7,4 & 3,3 & 1,7 & 2,7 & 1,6 & 1 & 0,9 & 88,9 \\
\hline Sul & 3,5 & 4,1 & 1,9 & 0,6 & 0,6 & 0,8 & 0,7 & 0,1 & 0,1 & 97,1 \\
\hline Leste & 1,6 & 4,5 & 2,2 & 0,6 & 0,4 & 0,7 & 0,8 & 0,2 & 0,2 & 87,5 \\
\hline Oeste & 43,6 & 51,5 & 20,2 & 7,6 & 5,4 & 5,5 & 5,1 & 4,3 & 2,5 & 94,3 \\
\hline Centro & 2,9 & 11,1 & 5,8 & 2,3 & 1,9 & 2,1 & 1,6 & 0,9 & 0,5 & 82,7 \\
\hline
\end{tabular}


Avaliação por Unidade Regional de Saúde. Em 1999, grande parte das URS apresentou municípios com elevada incidência da doença, destacando-se as regionais de Zé Doca, Pinheiro, Santa Inês e Açailândia, cuja população composta, em sua maioria, por lavradores, pescadores, trabalhadores do extrativismo vegetal, carvoeiros e madeireiros concentrava mais de 90\% dos casos de malária no Estado do Maranhão. 0 PIACM, iniciado em julho de 2000, conseguiu reduzir a incidência da malária nessas regionais na primeira avaliação programada para 2001. No entanto, em outras 11 URS, os índices se elevaram, 0 que não permitiu que 0 estado alcançasse a meta de redução da malária em 50\%. Dentre estas URS, destacam-se as regionais de Itapecuru-Mirim (incremento de 373,2\%), Bacabal (168,3\%), Rosário (107,8\%), Chapadinha (100\%) e Caxias (80\%).

Em 2004, houve elevação na incidência, com exceção das regionais de Açailândia, Bacabal e Santa Inês. Em 2005, somente quatro regionais apresentaram aumento da transmissão: Açailândia, Caxias, Presidente Dutra e Santa Inês. Ao final de 2007, somente as URS de Chapadinha, Itapecuru-Mirim e Presidente Dutra apresentaram incidências maiores, se comparadas a 1999, com variações de $16,6 \%, 52,1 \%$ e 80,7 , respectivamente (Tabela 2).

Unidades Regionais de Zé Doca e Pinheiro. Durante o período em estudo, as URS de Zé Doca e Pinheiro, áreas de alto e médio risco, apresentaram variações na incidência, sendo as duas responsáveis pelos maiores índices de malária do estado (Figura 3).

Zé Doca apresentou Incidência Parasitária de 79,8 e 75,2 por 1.000 habitantes em 1999 e 2000. Em 2001, a incidência foi de 29,3 (redução de 63,2\%), comparando-se a 1999. Apesar da redução da malária entre 1999 e 2007 ter sido significativa (90\%), esta se deu de maneira irregular com incrementos da incidência em 2004 (12,9/1.000) e $2006(16,8 / 1.000)$.

Pinheiro, em 1999 e 2000, teve incidências de 39,6 e 35,5/1.000. A redução da incidência entre 1999 e 2007 foi de 95,6\%. Comparando-se as duas regionais, esta teve redução menor na incidência em 2001 (25\%) e manteve maior oscilação em 2004 .

Unidades Regionais de Santa Inês e Açailândia. Nas URS de Santa Inês e Açailândia, áreas de Médio Risco, ao contrário das regionais anteriores, a incidência se elevou em 2000 (Figura 3).

Em Santa Inês, entre os anos de 1999 e 2000, a Incidência Parasitária Anual aumentou de 27/1.000 habitantes para 48,7/1.000. Em 2001, a incidência foi de 20,8/1.000 habitantes, apresentando uma redução de $22,9 \%$ quando comparado a 1999. Apesar da evolução ter sido significativa entre 1999 e 2007 (redução de 87,7\%), ocorreu de maneira irregular, com incrementos em 2005 e 2007.

Açailândia, após os anos de 1999 e 2000 (com incidências de 25,3 e 28,2/1.000 habitantes, respectivamente), apresentou redução, com algum incremento da transmissão em 2004.

Das quatro regionais em estudo, Açailândia com $98,1 \%$ foi a que apresentou maior índice de redução da malária.

Avaliação dos municípios incluídos no Plano de Intensificação das Ações de Controle da Malária. 0 Estado do Maranhão incluiu 46 municípios, o que representa 18\% dos 254 municípios amazônicos que ingressaram no PIACM.

Dos 27 municípios incluídos na região oeste, quatro eram de médio risco (entre 10 e 49 casos por 1.000 habitantes) e 23 de alto risco (mais de 50 casos por 1.000 habitantes). 0 índice de redução variou de 77,5\%, em Amapá do Maranhão, a 99,5\%,

TABELA 2

Incidência parasitária anual por Unidade Regional de Saúde, Estado do Maranhão, 1999 a 2007.

\begin{tabular}{|c|c|c|c|c|c|c|c|c|c|}
\hline \multirow[b]{2}{*}{ URS } & \multicolumn{9}{|c|}{ Ano } \\
\hline & 1999 & 2000 & 2001 & 2002 & 2003 & 2004 & 2005 & 2006 & 2007 \\
\hline Açailândia & 25,3 & 28,2 & 8,3 & 4,2 & 2,4 & 1,6 & 2,2 & 0,8 & 0,5 \\
\hline Bacabal & 6,0 & 26,9 & 16,1 & 6,3 & 4,6 & 4,6 & 2,4 & 1,9 & 1,1 \\
\hline Balsas & 0,2 & 0,6 & 0,1 & 0,04 & 0,1 & 0,2 & 0,1 & 0,03 & 0,02 \\
\hline Barra do Corda & 3,7 & 9,6 & 4,1 & 1,4 & 0,6 & 1,0 & 1,0 & 0,6 & 0,4 \\
\hline Caxias & 0,2 & 0,4 & 0,3 & 0,1 & 0,1 & 0,2 & 0,6 & 0,2 & 0,1 \\
\hline Chapadinha & 0,4 & 0,5 & 0,7 & 0,4 & 0,4 & 0,5 & 0,4 & 0,4 & 0,4 \\
\hline Codó & 6,6 & 20,1 & 9,1 & 1,8 & 1,2 & 1,9 & 1,9 & 1,0 & 0,5 \\
\hline Imperatriz & 5,0 & 5,7 & 1,7 & 0,8 & 0,9 & 1,1 & 0,9 & 0,7 & 0,4 \\
\hline Itapecuru & 0,7 & 7,1 & 3,4 & 2,4 & 0,7 & 2,2 & 0,4 & 0,6 & 1,1 \\
\hline Pedreiras & 1,0 & 1,5 & 1,2 & 0,6 & 1,3 & 1,4 & 1,3 & 1,3 & 1,0 \\
\hline Pinheiro & 39,6 & 35,5 & 26,7 & 7,9 & 5,7 & 8,9 & 5,9 & 1,6 & 1,7 \\
\hline Presidente Dutra & 0,3 & 0,9 & 0,3 & 0,6 & 0,9 & 0,9 & 1,8 & 0,6 & 0,5 \\
\hline Rosário & 7,6 & 26,9 & 15,8 & 10,7 & 3,5 & 5,5 & 2,4 & 0,9 & 1,0 \\
\hline Santa Inês & 27,0 & 48,7 & 20,8 & 5,9 & 2,6 & 2,5 & 3,5 & 3,0 & 3,3 \\
\hline São João dos Patos & 0,2 & 0,1 & 0,1 & 0,03 & 0,1 & 0,3 & 0,1 & 0,2 & 0,1 \\
\hline São Luís & 1,7 & 2,7 & 1,9 & 1,3 & 0,7 & 0,8 & 0,6 & 0,6 & 0,4 \\
\hline Viana & 5,9 & 13,6 & 7,3 & 2,5 & 1,1 & 2,5 & 0,8 & 0,9 & 0,5 \\
\hline Zé Doca & 79,8 & 74,2 & 29,3 & 12,7 & 11,8 & 12,9 & 10,2 & 16,8 & 8,0 \\
\hline
\end{tabular}

URS: Unidade Regional de Saúde. 


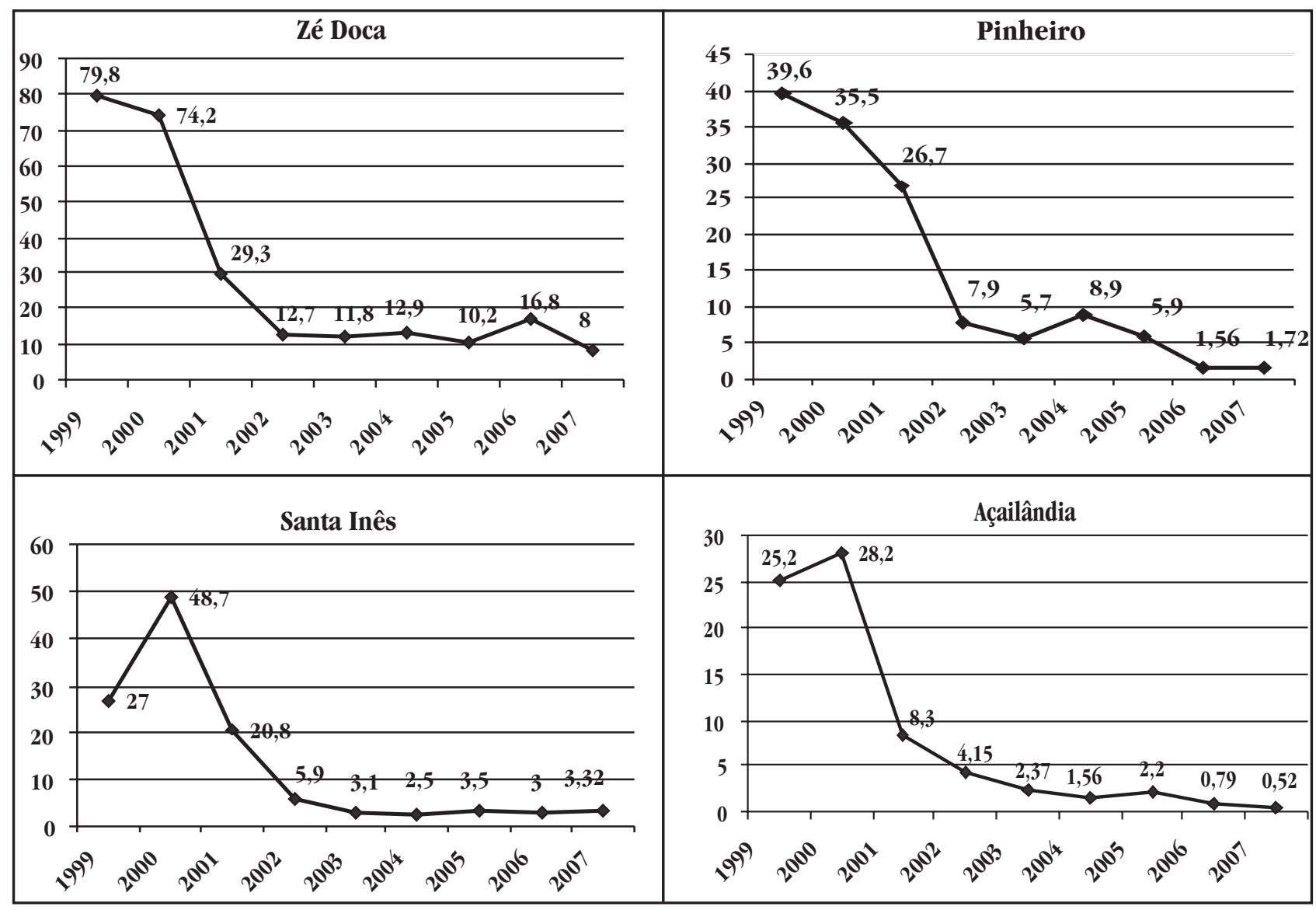

FIGURA 3

Evolução da incidência parasitária anual nas Unidades Regionais de Saúde de Zé Doca, Pinheiro, Santa Inês e Açailândia - Estado do Maranhão, 1999 e 2007.

em Bom Jesus das Selvas, todos municípios de alto risco. Dos 14 municípios incluídos da região norte, nove eram municípios de alto risco, inclusive o município de Conceição do Lago Açu, com a maior variação do índice de malária por Plasmodium falciparum do período (6,4\%, em 1999, para 59,7, em 2007). A região centro incluiu um município de alto risco, São Mateus do Maranhão, que tradicionalmente tinha a malária sob controle.

Ao final de 2007, dos 43 municípios considerados de alto e médio risco que participaram do PIACM, sete continuaram como médio risco. Os municípios de Centro Novo do Maranhão e Boa Vista do Gurupi, ambos da região oeste e na fronteira com o Estado do Pará, apresentaram as maiores incidências, respectivamente, 40,7 e 33,3 casos por mil habitantes. Com exceção de Alcântara, com 57,7\% e Centro Novo do Maranhão, com 69,4\%, os demais municípios obtiveram redução na incidência superior a $70 \%$.

\section{DISCUSSÃO}

Compreende-se que a estratégia adotada pela Conferência Ministerial de Amsterdam, introduzindo elementos novos, centrados na fonte de infecção e no controle integrado da malária, ajudou a mudar a visão campanhista ou vertical, oriunda da Campanha de Erradicação da Malária. Fortaleceu essa estratégia o processo de descentralização, em curso no Brasil, que atribuiu responsabilidades de saúde a estados e municípios.
Uma ligeira retrospectiva mostra que a malária constituía 0 maior problema de saúde pública no início do século XX, quando estava presente em 85\% do território nacional. Na década de 1940, seis milhões de casos anuais eram registrados em 70\% dos então 1.355 municípios brasileiros ${ }^{7119}$. Assim, no decorrer do século, diversas medidas de controle foram desenvolvidas, destacando-se a Campanha de Erradicação da Malária (CEM), implantada a partir de 1965. Baseada na estratégia de erradicação, preconizada pela Organização Mundial da Saúde (OMS), teve resultado positivo com a eliminação da malária das regiões Nordeste, Sudeste, Centro-Oeste e Sul.

Os casos foram reduzidos a 52.469, no início da década de 1970, distribuídos em uma área de 5,2 milhões de $\mathrm{km}^{2}$, formada pelos Estados do Amazonas, Pará, Acre, Rondônia, Roraima, Tocantins, Mato Grosso e Maranhão, possuidores de condições ambientais favoráveis à transmissão da doença e de uma realidade desafiadora, a ocupação desordenada do ambiente e presença de uma população migrante de garimpeiros, madeireiros e agricultores, vivendo em habitações precárias e em condições favorecedoras do fácil contato entre o mosquito transmissor e a fonte de infecção. Nessa situação, a escalada foi ascendente e o número de casos de malária chegou a 169.871 em 1980, o que levou ao emprego de novas iniciativas ${ }^{1619}$.

A iniciativa mais importante, ao nosso entender, foi a adotada em 1980 pela SUCAM, ao estratificar as áreas em alto, médio e 
baixo risco ${ }^{2}$ e nelas distribuir os recursos humanos, materiais e financeiros. Devido a dificuldades diversas, o registro de casos chegou a 577.520, no final da década, o que levou o governo brasileiro e recorrer ao Banco Mundial/BIRD para financiar um novo projeto, conhecido pela sigla PCMAM que vigorou de 1989 a 1995 e teve como principais objetivos reduzir a ocorrência de casos de malária; promover o desenvolvimento institucional da SUCAM e das Secretarias Estaduais de Saúde; fortalecer o controle da malária e atender a saúde da comunidade indígena ${ }^{619}$. 0 Plano de Controle da Malaria na Amazônia deu início à descentralização do controle da malária que teve continuidade com os planos seguintes ${ }^{348}$.

Assim como em outros estados amazônicos, o Maranhão, desde as décadas de 1970 e 1980, vem sofrendo o mesmo processo de expansão de suas fronteiras agrícolas, principalmente, nas regiões oeste e norte, pré-amazônicas e fronteiriças ao Estado do Pará, nas quais habita boa parte da população que depende da pesca, da lavoura, do extrativismo vegetal e vive de maneira itinerante em assentamentos improvisados e em condições de habitação e trabalho insatisfatórias. Nessas condições, a transmissão da malária se exacerbou alcançando, em 1986 e 1987, 54.668 e 56.627 casos, respectivamente, as maiores cifras registradas no estado e agravados pelos índices de Plasmodium falciparum (IFA), que alcançaram nos dois anos $55,5 \%$ e $54 \%$.

No primeiro ano de implantação do PIACM, na Região Amazônica, a partir de julho de 2000, o crescimento da malária continuou somente nos estados do Amapá, Pará e Maranhão, Neste último, estabeleceu-se um padrão de incidência que alcançou níveis epidêmicos em 32 dos 46 municípios incluídos no Plano, passando a contribuição do Maranhão à malária brasileira de $8,6 \%$ para $12 \%$, respectivamente, em 1999 e 2000 , cifras que se aproximaram das ocorridas na década de 1980.

No período de 1999 e 2007, alguns fatos relacionados ao controle da malária mostram que num estado com características geográficas e climáticas facilitadoras da transmissão e forte carga de suscetibilidade e vulnerabilidade, há necessidade de se implantar um sistema de vigilância cada vez mais eficaz como garantia da sustentabilidade dos planos para os anos seguintes. Por essa razão, as secretarias Estadual e Municipais de Saúde, devem organizar melhor seus serviços, para detecção precoce de casos e tratamento correto, praticar um eficiente controle vetorial e, dessa forma, cumprir os elementos técnicos preconizados pela Conferência de Amsterdam ${ }^{12}$ para controlar uma doença que, além do sofrimento individual, consome os escassos recursos dos municípios e do estado.

Os fatos observados foram: dificuldade de inserção da estratégia de saúde da família (PSF) e dos agentes comunitários nas ações de controle da malária em sua área de atuação; 0 surgimento da malária em municípios que tinham interrompido a transmissão, bem como a exacerbação da malária em municípios incluídos no Plano; a descontinuidade nas ações antivetoriais, a gradativa perda de informações sobre a suscetibilidade das espécies Anopheles darlingi e Anopheles aquasalis (nossos principais transmissores ${ }^{13}$ ) e, sobre o poder residual dos inseticidas utilizados na prática; o agravamento das manifestações clínicas da malária por Plasmodium vivax ${ }^{12}$, o plasmódio dominante no estado.

Outro fato, observado no período, foi o aumento na incidência da malária por Plasmodium falciparum (o IFA passou de 8,3\% para 37,5\% entre 1999 e 2007). Considerando a eficácia da terapêutica utilizada para esta espécie de plasmódio demonstrada pela redução dos indicadores de gravidade e de letalidade (25 óbitos em 1999 para dois em 2007) e o fato de que, nesta forma de malária, os gametócitos somente aparecem a partir do sétimo dia de doença, pode-se inferir que o aumento do IFA indica que a prática do diagnóstico e tratamento precoces não estão sendo devidamente obedecidas.

Apesar dos entraves apontados na política de controle da malária, nenhum insuperável, pode-se concluir, pelos números apresentados, que no Estado do Maranhão os planos foram eficazes entre 1999 e 2007, pois a redução na incidência da malária em $89,1 \%$ foi a maior entre todos os estados amazônicos.

\section{REFERÊNCIAS}

1. Barata RB. Malária no Brasil: panorama epidemiológico na última década. Cadernos de Saúde Pública 11:128-136, 1995.

2. Catão CPL, Mangabeira CJ, Tauil PL. Controle da malária no Brasil: 1965 a 2001. Revista Panamericana de Salud Publica 11:235-244, 2002.

3. Fundação Nacional de Saúde. Controle da Malária - Diretrizes Técnicas. Ministério da Saúde. Brasília, 1995.

4. Fundação Nacional de Saúde. Plano de Intensificação das Ações de Controle da Malária na Amazônia Legal - PIACM. Ministério da Saúde. Brasília, 2000.

5. Feitosa AC, Trovão JR. Atlas Escolar do Maranhão: Espaço Geo-histórico e Cultural. Editora Grafset, João Pessoa, 2006.

6. Ladislau JLB, Leal MC, Tauil PL. Avaliação do Plano de Intensificação das Ações de Controle da Malária na Região da Amazônia Legal, Brasil, no contexto da descentralização. Epidemiologia e Serviços de Saúde 15:9-20, 2006.

7. Marques AC, Cardenas H. Combate à malária no Brasil: evolução, situação atual e perspectivas. Revista da Sociedade Brasileira de Medicina Tropical 27:91-108, 1994.

8. Ministério da Saúde. Programa Nacional de Prevenção e Controle de Malária PNCM. Secretaria de Vigilância em Saúde, Brasília, 2003.

9. Ministério da Saúde. Malária. In: Guia de Vigilância Epidemiológica. 6a edição. Secretaria de Vigilância em Saúde, Brasília, p. 521-540, 2005.

10. Organización Mundial de la Salud. Aplicación de la Estrategia Mundial de Lucha Contra el Paludismo. Serie de Informes Tecnicos nº 839, Genebra, 1993.

11. Rachou RG. Atual estratégia da luta contra a malária. Revista Brasileira de Malariologia e Doenças Tropicais. Publicação avulsa nº 4, 1956.

12. Raposo CCBS. Malária vivax na Pré-Amazônia Maranhense: aspectos epidemiológicos e características clínicas. Tese de Mestrado, Universidade de Brasília, Brasília, DF, 2006.

13. Rebelo JMM. Manual de bio-ecologia dos vetores da malária. Universidade Federal do Maranhão, São Luís, MA, 2000.

14. Ribeiro MCT, Gonçalves EGR, Tauil P, Silva AR. Aspectos epidemiológicos de um foco de malária no município de São Luís, MA. Revista da Sociedade Brasileira de Medicina Tropical 38: 272-274, 2005.

15. Secretaria de Estado da Saúde. Gerência de Qualidade de Vida. Plano Estadual de Intensificação das Ações de Controle da Malária nos 46 Municípios do Estado do Maranhão da Amazônia Legal. Programa de Controle da Malária, São Luís, MA, 2000. 
16. Secretaria de Estado da Saúde. Plano de Controle Integrado da Malária nos 46 municípios do Estado do Maranhão. Programa Estadual de Controle da Malária, São Luís, MA, 2003.

17. Silva AR. Perspectiva de uma nova estratégia de controle da malária baseada na sua inserção no Sistema Único de Saúde (SUS). Tese de Concurso de Professor Titular. Universidade Federal do Maranhão, São Luís, MA, 1999.
18. Silva AR, Tauil PL, Bastos Jr JL, Matos WB, Costa EAPC, Gonçalves EGR. Aspectos da transmissão focal de malária na Ilha de São Luís, Maranhão. Revista da Sociedade Brasileira de Medicina Tropical 39:250-254, 2006.

19. Tauil PL. Avaliação de uma nova estratégia de controle da malária na Amazônia brasileira. Tese Doutorado, Universidade de Brasília, Brasília, 2002. 